\title{
Wolf-Rayet Stars
}

\author{
A. M. Cherepashchuk \\ Sternberg Astronomical Institute \\ Moscow University \\ 13, Universitetsky Prospect, 119899, Moscow, USSR
}

\section{INTRODUCTION}

Many new results have been obtained up to now in the problem of Wolf-Rayet (WR) stars. Most of these results are published now in the Proceedings of IAU Symposium $N 143$ (Van der Hucht and Hidayat, 1991), held in Baly in June 1990. Here we report some new additional results concerning mainly WR stars in binary systems.

\section{OBSERVATIONAL PROPERTIES OF THE BINARY WR STARS}

Recent results concerning the observational aspects of WR binaries have been summarized in the review by Cherepashchuk (1991). The characteristics of wellstudied WR+O binaries are listed, e.g., by Smith and Maeder (1989). Many masses are calculated using values of inclinations of the orbital plane $i$ determined from light curves and from polarization curves (see, e.g., Robert and Moffat, 1989).

The most important conclusions are the following.

2.1 The WR stars of all types are observed in WR+O systems: from WN3-WN7 to WC5-WC8 and WO4. One should note the absence of SB2 binaries among WN8,9 stars (Moffat, 1989)

2.2 The O-companions are ranging from $\mathrm{O} 4-\mathrm{O} 9.5$ and luminosity classes $\mathrm{V}-\mathrm{Ia}$. Among the WR stars, there are objects with composite spectra WN+WC, which may be suggested to be binary systems.

2.3 The mass ratio $q=\frac{m_{W R}}{m_{O}}$ for $\mathrm{WR}+\mathrm{O}$ binaries is ranging in the limits $0.17-$ $1.3-2.67$. The mean value is $q=0.44-0.53$ (here and below the second value is due to taking into account the system HD 92740 with $q \approx 2.67$ ). There is a good correlation between $q$ and spectral subtype $S p$ : the higher values of $q$ are observed for the latest $S p$ for WN and WC stars (Moffat at al., 1990).

2.4 Mean mass of WR stars, including WR stars in LMC and SMC (22 determinations), is $15.6-18.4 M_{\odot}$. The range of the value of the mass of WR stars is very wide: from $5 M_{\odot}$ to $48 M_{\odot}$ and even $77 M_{\odot}$ (HD 92740). Recent data allow to suggest that the masses of WC stars in average are less than those of WN stars, in agreement 
with the evolutional scenario for WR stars.

2.5 Mean mass for $\mathrm{O}$-companions in $\mathrm{WR}+\mathrm{O}$ binaries is $32.8 M_{\odot}$ and is ranging from 14 to $57 M_{\odot}$.

2.6 The masses of WR stars in $\mathrm{WR}+\mathrm{O}$ binaries are in average increasing with $q, S p$ and mass of the O-companion.

2.7 Mean value of the total mass of the $\mathrm{WR}+\mathrm{O}$ system is $48.5-51.5$ and is ranging in the limit $19-106 M_{\odot}$.

2.8 Known orbital periods for $\mathrm{WR}+\mathrm{O}$ systems are ranging from $1^{d} .6$ to $2886^{d}$.

2.9 Values of eccentricities of the orbit for $\mathrm{WR}+\mathrm{O}$ systems for all the systems with periods $P>70$ days are $e=0.3-0.8$. All the systems with the periods $P<14$ days have circular orbits $(e=0)$. In the interval $14^{d}<P<70^{d}$ there are both circular and eccentric orbits $(e=0.17-0.5)$ (see fig.1).

2.10 The luminosity of the bremsstrahlung $\mathrm{X}$-ray radiation $L_{x}$ in the $\mathrm{WR}+\mathrm{O}$ binaries (Pollock, 1987 ) correlates with the value of orbital period: $L_{x}$ is greater for the systems with shorter periods. This correlation is consistent with the theory of generation of X-ray radiation in the shock wave in front of the O-star (Cherepashchuk, 1967, 1976, Prilutsky and Usov, 1976, Stevens et al., 1991).

2.11 About half of the well-investigated $\mathrm{WR}+\mathrm{O}$ binaries are eclipsing with the amplitude of photometric variability $\Delta V=0^{m} .025-0^{m} .5$. Variable linear polarization (up to $0.5 \%$ ) for many of these $\mathrm{WR}+\mathrm{O}$ systems has been observed recently and the values of $i$ and $\dot{M}$ determined (e.g. St-Louis at al, 1988).

New data on WR+O binaries support the basic idea that WR stars are bare cores of massive stars being at a late evolutionary stage (Paczynski, 1973, Tutukov and Yungelson, 1973, Conti, 1976, Van den Heuvel, 1976, Kornilov and Lipunov, 1983, Vanbeveren, 1991, Maeder, 1991).

This conclusion is supported also by recent theoretical and observational data for single WR and $\mathrm{O}$ stars as well as for some peculiar objects (LBV, RY Sct, SS 433, supernova remnants of CasA-type).

In particular, investigations of WR stars in SMC, LMC and several galaxies of the Local Group (M 31, M 33, NGC 6822, IC 1613, IC 4662) revealed the observed number frequency of WR stars in connection between the local metallicity $Z$ and mass loss (see e.g. Maeder, 1991 and all references therein).

New spectral analyses of WR stars based on advanced multi-level non-LTE model of extended stellar atmosphere (e.g. Hamman, 1985, Hillier, 1988, Schmutz et al., 1989, Hamman et al., 1991, Kudritsky and Hummer, 1990 and all references therein) provide growing evidence that the basic parameters of WR stars (core radius, core temperature, $\dot{M}$, chemical composition) are drastically different from those of normal hydrogen-burning stars. Such an evidence has been obtained also from the analysis of the eclipsing WR binary systems (e.g. Cherepashchuk, 1982, Cherepashchuk et al., 1984).

Let us consider some specific observational effects which are of great interest for 
understanding the WR phenomenon.

\section{THE ENHANCED OPACITY OF WR WIND IN UV RANGE; SELECTIVE ATMOSPHERIC ECLIPSES}

The effect of enhanced opacity in WR wind has been discovered by Eaton et al. $(1982,1985 \mathrm{a}, \mathrm{b})$ for the ultraviolet region of the spectrum at $\lambda<2000 \AA$ using IUE observations of WR eclipsing binary systems V 444 Cyg and CV Ser. This effect is observed also in other galactic WR binaries (Hutchings and Massey, 1983, Koenigsberger and Auer 1985). The depth of atmospheric eclipse strongly increases in the range $\lambda<1500 \AA$; this is caused by the absorption of the O-star light in many lines of ions FeIV, FeV and FeVI (Koenigsberger, 1988). In the atmospheric eclipse of HD 5980, Koenigsberger et al. (1987) did not find the increased absorption at $\lambda \lambda 1300-1500 \AA$; this reflects a relatively poor abundance of heavy elements in $\mathrm{SMC}$. Ions of FeIV, FeV, FeVI have the ground state with the electron configuration $3 d^{n+1}$ and a relatively low-energy metastable state $3 d^{n} 4 s$. Transitions between the many states $3 d^{n} 4 s$ and the upper state $3 d^{n} 4 p$ leads to the formation of a large number of permitted absorption lines (Bowen, 1937, Ekberg, 1975). The limits of the series for $\mathrm{FeV}(\lambda 1560 \AA)$ and FeIV are close to the spectral range in which the enhanced opacity is observed in the spectra of WR stars. The exitation of the metastable levels of the ions of Fe may be provided by the radiation mechanism by the absorption of the EUV radiation of the hot WR core $(\sim 500 \AA)$ with the transition $3 d^{n+1} \rightarrow 3 d^{n} 4 p$ and the following decay in $3 d^{n} 4 s$. In an atmospheric eclipse by a WR star these lines would be spread out into a continuum by the velocity dispersion through the atmosphere, leading to the absorption in the quasicontinuum. Bruhweiler et al. (1981) presented a list of such lines in the spectrum of the O-type subdwarf HD 4978, Nugis and Sapar (1985) showed from high-resolution IUE spectra of the WN6 star HD 192163 that ions of FeV and FeVI are dominating in the WR spectrum at $\lambda<1720 \AA$. Another explanation of the source of UV extinction in the range $\lambda<2000 \AA$ for a WR atmosphere has been proposed by Bochkarev (1985): scattering in the wings of strong UV resonant lines of abundant elements (C, N, O, Si, etc.). Since in the spectrum HD 5980 the effect of enhanced absorption for $\lambda<2000 \AA$ is not observed (Koenigsberger et al., 1987), the mechanism proposed by Bochkarev(1985) can be applied for EUV range of the spectrum of WR stars but not for the range 1100$2000 \AA$.

The theory of selective atmospheric eclipses in WR+O binary systems has been developed by Khaliullin and Cherepashchuk (1976). For the first time, selective atmospheric eclipses were discovered in the WR+O system CV Ser (Cherepashchuk, 1969, $1971 \mathrm{a}, 1971 \mathrm{~b})$ in the emission band CIII-CIV $4653 \AA$ (fig.2). The emission line CIIICIV $4650 \AA$ corresponds to the transition $3^{3} P^{0}-3^{3} S$ where the state $3^{3} S$ is metastable. This state must be overpopulated due to the dilution effect in an extended WR at- 
mosphere, leading to the enhanced opacity of the WR envelope for the great distance from the WR core: up to $30 r_{\text {core }}$.

It should be noted that for the first time the idea about overpopulation of the metastable states of atoms due to the dilution effects was proposed by Ambartsumian (1933). This idea has been developed by Struve and Wurm(1938), Wellman (1951),Mathis (1957) Ghobros (1962), Underhill (1966). Enhanced opacity of outer parts of the WR envelope due to the dilution effects allows us to investigate the structure of WR wind for the great distance from WR core where the opacity of matter due to the electron scattering is negligible (Khaliullin and Cherepashchuk, 1976, Koenigsberger,1990). Enhanced opacity of WR wind in the UV range must be taken into account when the temperature of the WR core is determined from UV data. This enhanced opacity of matter due to the overpopulation of metastable states of the atoms of heavy elements may be important also for the interpretation of the spectra of the nuclei of Seyfert galaxies and quasars. In the case of quasars, due to a high value of redshift, the effects of enhanced opacity of the matter can be observed in the optical range.

\section{BLOB STRUCTURE OF WR WIND}

A WR star is seen through an extended atmosphere, due to strong wind: $\dot{\mathrm{M}}$ $\sim 10^{-4}-10^{-5} M_{\odot} /$ year. So, the observed characteristics of WR stars are strongly dependent on the properties of their winds. The new three-dimensional spectral classification of WR stars (Schmuz et al., 1989, Hamman et al., 1991) based on advanced multi-level non-LTE model of extended atmosphere depends on the model of wind.

Recently, there were obtained growing evidences for the inhomogeneous, blob structure of WR wind.

\subsection{Analysis of infrared eclipses in WR+O binary systems}

Width and depth of the eclipse of the WR star by the O6 star in the binary system V $444 \mathrm{Cyg}$ (WN5+O6, $p \approx 4^{d} .2$ ) are strongly increasing with wavelength (Cherepashchuk et al., 1984). This effect has been confirmed by new infrared observations of V444 Cyg (Cherepashchuk et al., 1991) -- see fig.3. Restoration of brightness distribution over the WR disk from these data (Cherepashchuk et al., 1984) allows us to conclude that the dimensions of the envelope of the WR star are strongly increasing with $\lambda$ (see fig.4). This increase cannot be explained by the new theory of the extended WR atmosphere (e.g. Hamman et al., 1991) in the framework of the standard model of homogeneous WR wind described by Lamers velocity law (Schmutz, 1990). Also the densities in the atmospheric regions of low electron - scattering optical depth are significantly lower than those found by Hartmann(1978) by 
means of fitting the optical and infrared secondary eclipses in the V 444 Cyg system, using an atmospheric model for a WR star. As was pointed out by Cherepashchuk et al.(1984), this discrepancy results from the effect of density fluctuations (blob structure) in the wind on the infrared opacity. Since in the infrared range the main absorption mechanism is free-free absorption, the emissivity and absorption properties of matter of the WR envelope in the infrared range are proportional to $n_{e}^{2}$. Non linear dependence of properties of the WR envelope on electron density $n_{e}$ leads to a strong increase of the dimensions of the WR envelope in the infrared range in the case of blob structure of WR wind. In this connection, it would be very interesting to obtain infrared light curves for other WR eclipsing binaries, especially HD 5980 in SMC.

\subsection{Deficiency of $\mathrm{X}$-ray radiation from shock waves in short-period $\left(p<20^{d}\right)$ binary WR+O systems.}

The theoretical value of the $\mathrm{X}$-ray flux from a shock wave formed in front of the $\mathrm{O}$ star by supersonic flow of WR wind (Cherepashchuk, 1967, 1976, Prilutsky and Usov, 1976, Bajramov et al.,1990, Stevens et al, 1991) is by order of magnitude less than the observed one (Pollock, 1987). This fact may be understood in the framework of the model of blob wind: dense clouds of matter (density jump $\sim 10-100$ ) penetrate easily through the shock wave formed by the continuous component of WR wind in front of the O-star and are decelerated in the photosphere of the O-star where the $\mathrm{X}$-ray radiation is transformed into the optical-range one (Cherepashchuk, 1990). Up to $80 \%$ of mass loss of the WR star is ejected as a number of dense and compact clouds of matter (Cherepashchuk, 1990).

\subsection{Microvariability of the emission lines in the spectra of WR stars.}

High-resolution spectral observations of single WR stars carried out with a very high signal-to-noise ratio $(\sim 300)$ revealed the fine variable structure on the top of the emission line profiles HeII 5411 (Moffat et al., 1991) and NIV 4058, HeII 4200, CIV 4653 (Vreux et al, 1991, Moffat and Robert, 1991). Sometimes the regular motions of the individual narrow peaks on the top of the emission lines are observed (Moffat et al.,1988). These facts may be considered as a direct evidence for the blob structure of WR wind.

\subsection{Period changes in WR+O eclipsing binaries V 444 Cyg, CQ Cep.}

The value of the mass loss rate $\dot{M}$ for WR star from the observed period changes in WR+O eclipsing binaries (Khaliullin, 1974, Kornilov and Cherepashchuk, 1979, Khaliullin et al,1984, Antokhina et al. 1982, Underhill et al., 1990) $\dot{M}=(0.6-$ 1) $10^{-5} \mathrm{M}_{\odot} /$ year is $2-3$ times less than that derived for the same WR stars from IR and 
radio observations (e.g. Willis, 1991). In the case of spherical symmetry, supposing homogeneous structure of stellar wind, the formula developed by Wright and Barlow (1975) may be used to determine the mass loss rate $\dot{M}$ from radioobservations of WR stars:

$$
\dot{\mathrm{M}}=0.095 v_{\infty} \frac{S_{\nu}^{0.75} D^{1.5}}{(g \nu)^{0.5}} \frac{\mu}{z \gamma^{0.5}} M_{\odot} / \text { year }
$$

where $S_{\nu} \sim n_{e}^{2} f\left(T_{e}\right)$ - is the radio flux (Jy) at the frequency $\nu(\mathrm{Hz}) ; v_{\infty}(\mathrm{km} / \mathrm{s})$; $\mathrm{D}$ - the distance (kpc); $\mathrm{g}$ is the Gaunt factor; $\mu, z$ and $\gamma$ are, respectively, the mean molecular weight, r.m.s. ionic charge and mean number of electrons per ion. In the radio region the free-free spectrum is predicted to have a distribution $S_{\nu} \sim \nu^{\alpha}$ where $\alpha=0.6$. Non linear dependence of $S_{\nu}$ on the electron density $n_{e}$ leads to the increasing of the $S_{\nu}$ for the same $\dot{M}$ in the case of blob structure of WR wind. Thus the blob structure of WR wind may be responsible for the discrepancy between the value of $\dot{M}$ derived from the period changes of the eclipsing $\mathrm{WR}+\mathrm{O}$ binaries and $\dot{M}$ determined from radioobservations. Therefore, if the blob structure of WR wind exists at the distance up to $10^{15} \div 10^{17} \mathrm{~cm}$ from the WR star, the values of $\dot{M}$ derived from IR and radio observations may be overestimated.

\subsection{Deficiency of X-ray radiation from the ring nebula NGC 6888 surrounding the WR star HD 192163.}

The observed X-ray luminosity of the ring nebula NGC 6888 formed by stellar wind of the WR star $L_{x} \sim 10^{32} \mathrm{erg} / \mathrm{s}$ (Bochkarev, 1988) is by an order of magnitude less than the one predicted from the theoretical grounds in the framework of the homogeneous model of WR wind (Pikelner and Scheglov, 1968). It may be considered as an evidence for cloud structure of WR wind at the distances of about $10^{17}-10^{18}$ $\mathrm{cm}$ from the WR star.

\subsection{Irregular microvariability of the intensity and linear polarization of the light of WR stars.}

Such a microvariability has been discovered recently for many single WR stars and may be connected with the instability and blob structure of WR wind (Lamontagne and Moffat, 1987, Moffat, 1988, van Genderen et al., 1987). Increasing of the amplitude of the microvariability of WR stars with the decreasing of the velocity of WR wind has been pointed out (e.g. Moffat, 1988).

\subsection{Theoretical investigations of the instability of stellar winds.}

Stellar wind driven by radiation may be instable (Owocki and Rybicki, 1984, 1990, Owocki et al., 1988, Owocki, 1991). Although including scattering effects might 
reduce, or even break steady-state solution degeneracy of line-driver stellar wind, the relatively small photon destruction probability can cause a flow with a large continuum optical thickness (this is the case of WR wind) to remain instable (Owocki and Rybicki, 1990).

\section{PROFILES OF THE EMISSION LINES HEI AND HEII IN THE FRAMEWORK OF THE MODEL OF BLOB STRUCTURE OF WR WIND}

These profiles have been calculated by Antokhin et al.(1992) under the following suppositions: the wind of the WR star is spherically symmetric, all clouds originate at the same distance $r_{o}$ from the WR core; clouds are spherically symmetric, the mass of the cloud is constant, the number of clouds is large $\left(>10^{4}\right)$ and is described by the macroscopic parameter $\delta(r)$ - filling factor. Solution of the stationary equations for the intercloud matter has been carried out by Sobolev's method (Sobolev, 1960, Castor, 1970), for the cloud matter - by the modified Sobolev's method (Boland and de Jong, 1984). The profiles of the lines HeI 5876 and HeII 5411 for the WN5 star HD 191765 have been calculated. Observed profiles of these lines have been taken from the paper of Underhill et al.(1990). The best agreement was obtained for the following parameters of the WR star: $\dot{M}=2 \cdot 10^{-5} M_{\odot} /$ year, $T_{\text {core }}=60000 \mathrm{~K}$, $\bar{T}_{e}=30000 \mathrm{~K}, r_{\text {core }}=3 R_{\odot}, v_{0}=200 \mathrm{~km} / \mathrm{s}$ (velocity of wind at the surface of the core of the WR star), $v_{\infty}=2200 \mathrm{~km} / \mathrm{s}, \beta=0.6$ (parameter of the Lamers velocity law), $r_{0}=3.5 R_{\odot}$ (radius of the formation of the clouds), $\delta=0.035$ (initial filling factor for the clouds), $p_{0}=40$ (initial density jump in the cloud), $r_{c l}^{o}=0.1 R_{\odot}$ (initial radius of the cloud), $v_{c l}^{e x p}=30 \mathrm{~km} / \mathrm{s}$ (velocity of the expansion of the cloud); the portion of the mass loss rate carried by the clouds is $\sim 59 \%$. The comparison of the theoretical and observed emission line profiles is shown at the fig.5. The agreement between the observations and theory is quite the same as has been obtained by Schmutz et al.(1989), but we described the observed spectrum of HD 191765 with the value of mass loss rate $\dot{M}=2 \cdot 10^{-5} M_{\odot} /$ year which is 5 times less than that obtained by Schmutz et.al.(1989). This is due to using the cloud structure model for WR wind in our case. There is some discrepancy between theory and observations for the emission line HeI 5876. For the explanation of the peculiar shape of the profile of the HeI 5876 line, Underhill et.al.(1990) proposed the disc-like model for the extended atmosphere of the WR star. Some observational and theoretical grounds for the rapid rotation of the WR cores appeared recently (e.g.Van Genderen et al., 1987, Poe et al., 1989, Schulte-Ladbeck et al., 1990, Vreux et al., 1991). But the outer emission-line forming region of WR envelope remains quasispheric because there is no correlation between the widths of the emission lines of WR stars and the inclination of their rotational axes to the line of sight (Aslanov and Cherepashchuk, 1990). By introducing some local asymmetry into our blob model of WR wind (non-sphericity of the individual clouds 
due to their inhomogeneous expansion), we achieved a good agreement between the observational and theoretical profiles of the emission line HeI 5876 (see fig.7).

From our investigations of the blob structure of WR wind, two important conclusions may be drawn:

a) Due to blob structure of the WR wind, new three-dimensional spectral classification of WR stars, based on the advanced multi-level non-LTE model of a homogeneous extended atmosphere (Schmutz et al., 1990) may be not unique.

b) Since the blob structure of stellar wind leads to a lower value of mass loss rate $\dot{M}$, the observed number frequency of WR stars in connection between the local metallicity $Z$ and mass loss rate (Maeder, 1991) may be reanalysed. In particular, the binary evolution may be important for the formation of the WN and WC stars.

Recent results, concerning $\mathrm{WR}+\mathrm{C}$ binary systems containing possible relativistic object, are presented in the review by Cherepashchuk (1991). Some correlation between the amplitude of the optical microvariability $\Delta \mathrm{V}$ and the spectral type $\mathrm{Sp}$ of the WR star has been suggested (Cherepashchuk, 1991), which may be considered as an argument against the binary nature of the majority of the suspected $\mathrm{WR}+\mathrm{C}$ binaries. Nevertheless, the search for relativistic companions in WR binary systems remains a very important problem.

\section{REFERENCES}

Ambartsumian, V.A. 1933, Circular of Pulkovo Obs. N 6, 10.

Antokhin, I.I., Nugis, T., Cherepashchuk, A.M. 1992, Astron.Zh.(USSR) 69, in press.

Antokhina, E.A., Lipunova, N.A., Cherepashchuk, A.M. 1982, Astron.Zh.(USSR) 59, 704 ,

Aslanov, A.A., Cherepashchuk, A.M. 1990, Astron.Zh.(USSR) 67, 1195.

Bajramov, Z.T., Pilyugin,N.N., Usov, V.V. 1990, Astron.Zh.(USSR) 67, 998.

Bochkarev, N.G. 1985, in: R.M.Hjellming, D.M.Gibson (eds), Radio Stars, D.Reidel, p. 121.

Bochkarev, N.G. 1988, Nature, 332, 518.

Boland, W., de Jong, T. 1984, Astron.Astrophys. 134, 87.

Bowen, I.S. 1937, Phys.Rev. 52, 1153.

Bruhweiler, F.C., Kondo, Y., Mc Cluskey, G.E. 1981, Astrophys. J. Suppl. 46, 255.

Castor, J.I. 1970, Monthly Notices Roy. Astron. Soc. 149, 111.

Cherepashchuk, A.M. 1967, Variable Stars (USSR) 16, 226.

Cherepashchuk, A.M. 1969, Astron. Tzirk.(USSR) N 509.

Cherepashchuk, A.M. 1971a, Astron. Tzirk.(USSR) N 602.

Cherepashchuk, A.M. 1971b, Astron.Zh.(USSR) 48, 1201 (Sov.Astron. 15, 955).

Cherepashchuk, A.M. 1976, Pis'ma Astron.Zh.(USSR) 2, 356 (Sov.Astron.Letters 2, 138)

Cherepashchuk, A.M. 1982, Astrophys. Space Sci. 86, 299. 
Cherepashchuk, A.M. 1990, Astron.Zh.(USSR) 67, 955 (Sov.Astron. 34, 481).

Cherepashchuk, A.M. 1991, in: K.A. Van der Hucht, B.Hidayat (eds) Wolf-Rayet Stars and Interrelations with other Massive Stars in Galaxies, IAU Symp. N 143 (The Netherlands), p. 187.

Cherepashchuk, A.M., Eaton, J.A., Khaliullin, Kh.F. 1984, Astrophys. J. 281, 774.

Cherepashchuk, A.M. et al. 1992, Astron.Zh.(USSR) 69, in press.

Conti, P. 1976, Mem. 8 Soc. R. Sci. Liege (6) 9, 193.

Eaton, J.A., Cherepashchuk, A.M., Khaliullin, Kh.F. 1982, in: Y.Kondo, J.M.Mead and R.D.Chapman (eds.), Advances in Ultraviolet Astronomy: Four Years of IUE Research, NASA CP-2238, p.542.

Eaton, J.A., Cherepashchuk, A.M., Khaliullin, Kh.F. 1985a, Astrophys. J. 296, 222.

Eaton, J.A., Cherepashchuk, A.M., Khaliullin, Kh.F. 1985b, Astrophys. J. 297, 266.

Ekberg, J.O. 1975, Phys. Scripta 11, 23.

Ghobros, R. 1962, Z. Astrophys. 56, 113.

Hamman, W.R. 1985, Astron. Astrophys. 148, 364.

Hamman, W.R., Dünnebeil, G., Koesterke, L., Schmutz, W., Wessolowski, U. 1991, Astron. Astrophys., in press.

Hartmann, L. 1978, Astrophys. J. 221, 193.

Hillier, D.J. 1988, Astrophys. J. 327, 822.

Hutchings, J.B., Massey, P. 1983, PASP 95, 151.

Khaliullin, Kh.F. 1974, Soviet Astr. 18, 229.

Khaliullin, Kh.F., Cherepashchuk, A.M. 1976, Astron.Zh.(USSR) 53, 327 (Soviet Astr. 20, 186).

Khaliullin, Kh.F., Khaliullina, A.I., Cherepashchuk, A.M. 1984, Pis'ma Astron.Zh. (USSR) 10, 600.

Koenigsberger, G. 1988, Revista Mexicana Astron.Astrof. 16, 75.

Koenigsberger, G. 1990, Astron. Astrophys. 235, 282.

Koenigsberger, G., Auer, L.H. 1985, Astrophys. J. 297, 255.

Koenigsberger, G., Moffat, A.F.J.,Auer, L.H. 1987, Astrophys. J. 322, L41.

Kornilov, V.G., Cherepashchuk, A.M. 1979, Pis'ma Astron.Zh. (USSR) 5, 398.

Kornilov, V.G., Lipunov, V.M. 1983, Astron.Zh.(USSR) 60, 284, 754.

Kudritsky, R.P., Hummer, D.G. 1990, Annual Rev. of Astron. Astrophys. 28, 303.

Lamontagne, R., Moffat, A.F.J. 1987, Astron. J. 94, 1008.

Maeder, A. 1991, Astron. Astrophys. 242, 93.

Mathis, J. 1957, Astrophys. J. 125, 318.

Moffat, A.F.J. 1988, in: Polarized Radiation of CS Origin (Vatican), p.607.

Moffat, A.F.J. 1989, Astrophys. J. 347, 373.

Moffat, A.F.J.,Robert, C. 1991, in: K.A. Van der Hucht, B.Hidayat (eds) Wolf-Rayet Stars and Interrelations with other Massive Stars in Galaxies, IAU Symp. N 143 (The Netherlands), p. 109.

Moffat, A.F.J., Drissen, L., Lamontagne, R., Robert, C. 1988, Astrophys. J. 334, 
1038.

Moffat, A.F.J., Drissen, L., Robert, C., Lamontagne, R., Coziol, R., Mousseau, N., Niemela, V.P., Cerruti, M.A., Seggewiss, W., Van Weeren, N. 1990, Astrophys. J. 350, 767.

Nugis, T., Sapar, A. 1985, High-Resolution Spectrum of the WN6 Star HD 192163: the FeV and FeVI Dominated Emission line Spectrum at $\lambda \leq 1720 \AA$, Tallinn, Valgus.

Owocki, S.P. 1991, in: K.A. Van der Hucht, B.Hidayat (eds) Wolf-Rayet Stars and Interrelations with other Massive Stars in Galaxies, IAU Symp. N 143 (The Netherlands), p. 155.

Owocki, S.P., Rybicki, G.B. 1984, Astrophys. J. 284, 337.

Owocki, S.P., Rybicki, G.B. 1990, Astrophys. J.,in press.

Owocki, S.P., Castor, J.I.,Rybicki, G.B. 1988, Astrophys. J., 335, 914.

Paczynski, B. 1973, in: M.Barry, J.Sahade (eds.) Wolf-Rayet and High Temperature Stars, IAU Symp. N 49, (Dordrecht, D.Reidel), p.143.

Pikelner, S.B., Scheglov, P.V. 1968, Astron.Zh. (USSR) 45, 953.

Poe, C.H., Friend, D.B., Cassinelli, J.P. 1989, Astrophys. J., 337, 888.

Pollock, A.M.T. 1987, Astrophys. J., 320, 283.

Prilutsky, O.F., Usov, V.V. 1976, Astron.Zh. (USSR) 53, 6.

Robert, C., Moffat, A.F.J. 1989, Astrophys. J., 343, 902.

Schmutz, W. 1990, private communication.

Schmutz, W., Hamman, W.R., Wessolowski, U. 1989, Astron. Astrophys. 210, 236.

Schulte-Ladbeck, R.E., Nordsieck, K.N., Nook, M.A., Magalchäes, A.M., Taylor, M., Bjorkman, K.S., Anderson, C.M. 1990, Astrophys. J. 365, L19.

Smith, L.F., Maeder, A. 1989, Astron. Astrophys. 211, 71.

Sobolev, V.V. 1960, Moving Envelopes of stars, (Cambridge: Harvard University Press).

Stevens, I.R., Blondin, J.M., Pollock, A.M.T. 1991, Astrophys. J., in press.

Struve, O., Wurm, K. 1938, Astrophys. J. 88, 84.

St.-Louis, N., Moffat, A.F.J., Drissen, L., Bastein, P., Robert, C. 1988, Astrophys. J. 330, 286.

Tutukov, A.V., Yungelson, L.R. 1973, Nauchn. Inform. Asrtrosov. A.N.USSR 27, $58,70,86$.

Underhill, A.B. 1966, The Early Type Stars, (D.Reidel, Dordrecht, Holland).

Underhill, A.B., Grieve, G.R., Louth, H. 1990a, PASP 102, 749.

Underhill, A.B., Gilory, K.K., Hill, G.M., Dinshaw, N. 1990, Astrophys. J. 351, 666.

Vanbeveren, D. 1991, Space Sci. Rev. 56, 249.

Van den Heuverel, E.P.J. 1976, in: P.P.Eggleton et.al.(eds.) Structures and Evolutions of Close Binary Systems (Dordrecht, D.Reidel), p.35.

Van der Hucht, K.A., Hidayat, B. (eds.) Wolf-Rayet Stars and Interrelations with other Massive Stars in Galaxies, IAU Symp. N 143 (The Netherlands). 
Van Genderen, A.M., Van der Hucht, K.A., Steemers, W.J.G. 1987, Astron. Astrophys. 185, 131.

Vreux, J.M., Gosset, E., Bohannan, B., Conti, P., 1991, Astrophys. J., in press.

Wellman, P., 1951, Z. Astroph. 30, 71.

Willis, A.J., 1991, in: K.A. Van der Hucht, B.Hidayat (eds) Wolf-Rayet Stars and Interrelations with other Massive Stars in Galaxies, IAU Symp. N 143 (The Netherlands), p. 265

Wright, A.E., Barlow, M.J. 1975, Monthly Notices Roy. Astron. Soc. 170, 41. 

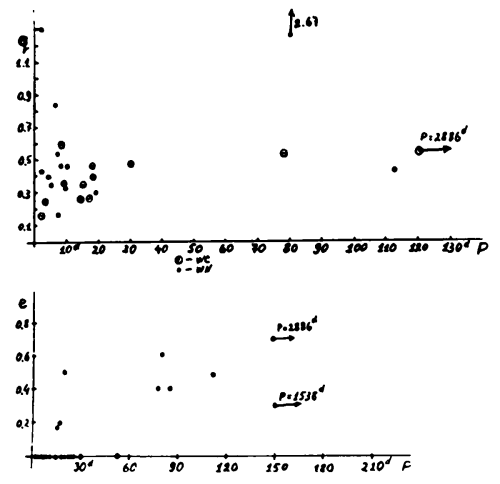

Fig.1 Top: the dependence of mass ratio $q=M_{W R} / M_{O}$ in $\mathrm{WR}+\mathrm{O}$ binary systems on the value of the orbital period $P$. Bottom: the dependence of the eccentricity $e$ of the orbit for $\mathrm{WR}+\mathrm{O}$ binaries on the orbital period $P$.

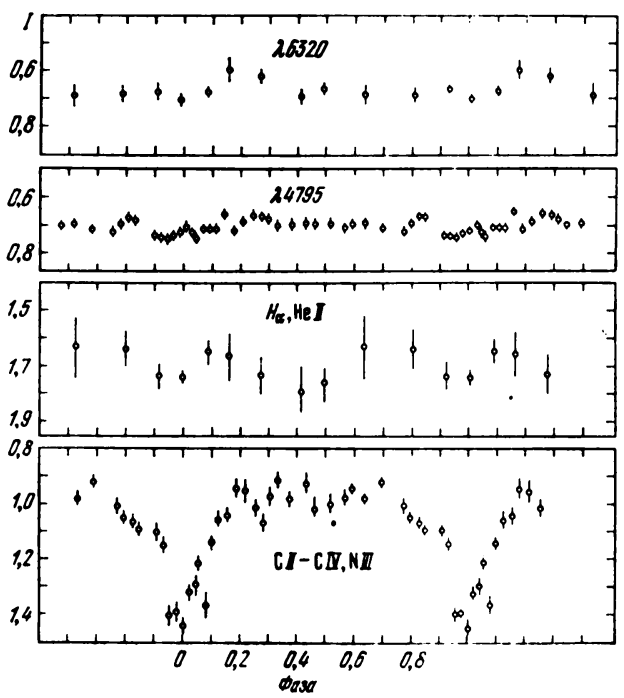

Fig.2 Average light curves of the WR+O binary CV Ser in the continuum $\lambda 6320 \AA$, $\lambda 4795 \AA$ and in the emission lines $\mathrm{H}_{\alpha}$, HeII and CII-CIV, NIII $4653 \AA$ (Cherepashchuk, 1969, 1971 a,b). 


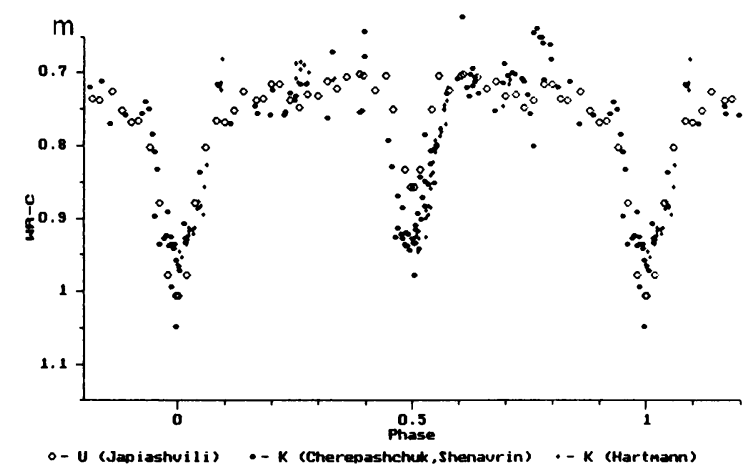

Fig.3 Comprasion of the optical U-light curve of V 444 Cyg (cirsles) and infrared K-light curve (points). The U-light curve is obtained by E.B.Japiashwili, K-light curve - by A.M.Cherepashchuk and V.Shenavrin.

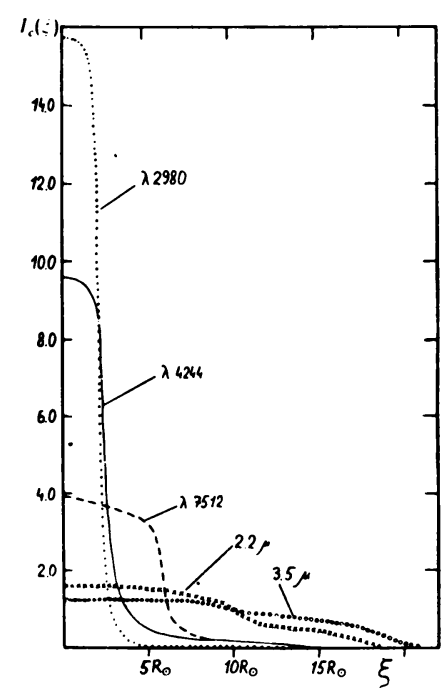

Fig.4 Brightness distributions over the disk of the WN5 star in the V 444 Cyg system restored by solution of the light curves in the secondary minimum for different wavelengths $\lambda$. 


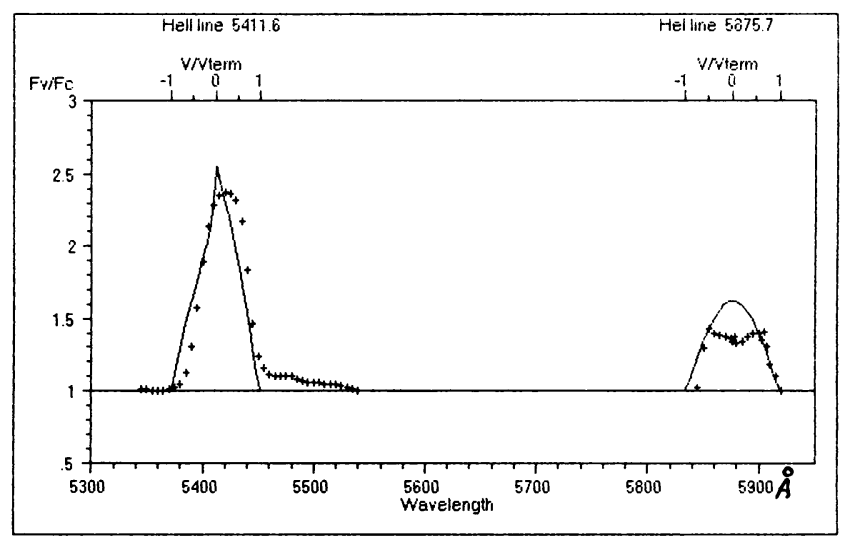

Fig.5 Theoretical and observed (crossed) profiles of the emission lines Hel $5875.7 \AA$ and HeII $5411.6 \AA$ for the WN5 star HD 191765. Theoretical profiles are calculated in the framework of the blob structure of WR wind, the blobs are sherical.

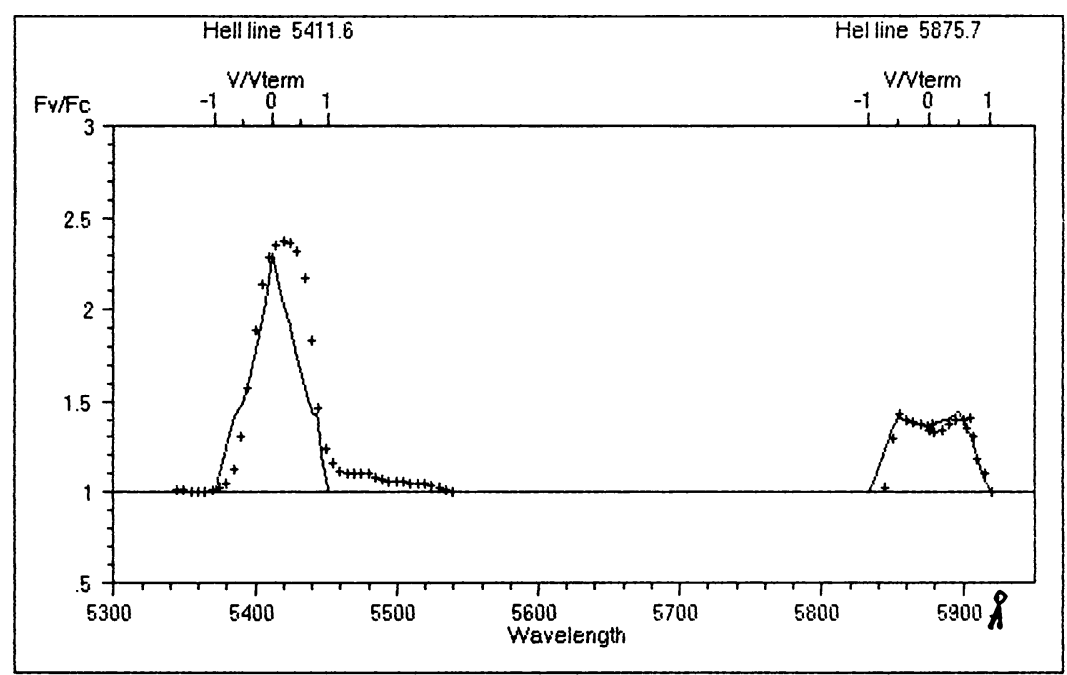

Fig.6 The same as at the fig.5. Non-sphericity of the individual blobs due to their inhomogeneous expansion is taken into account. 